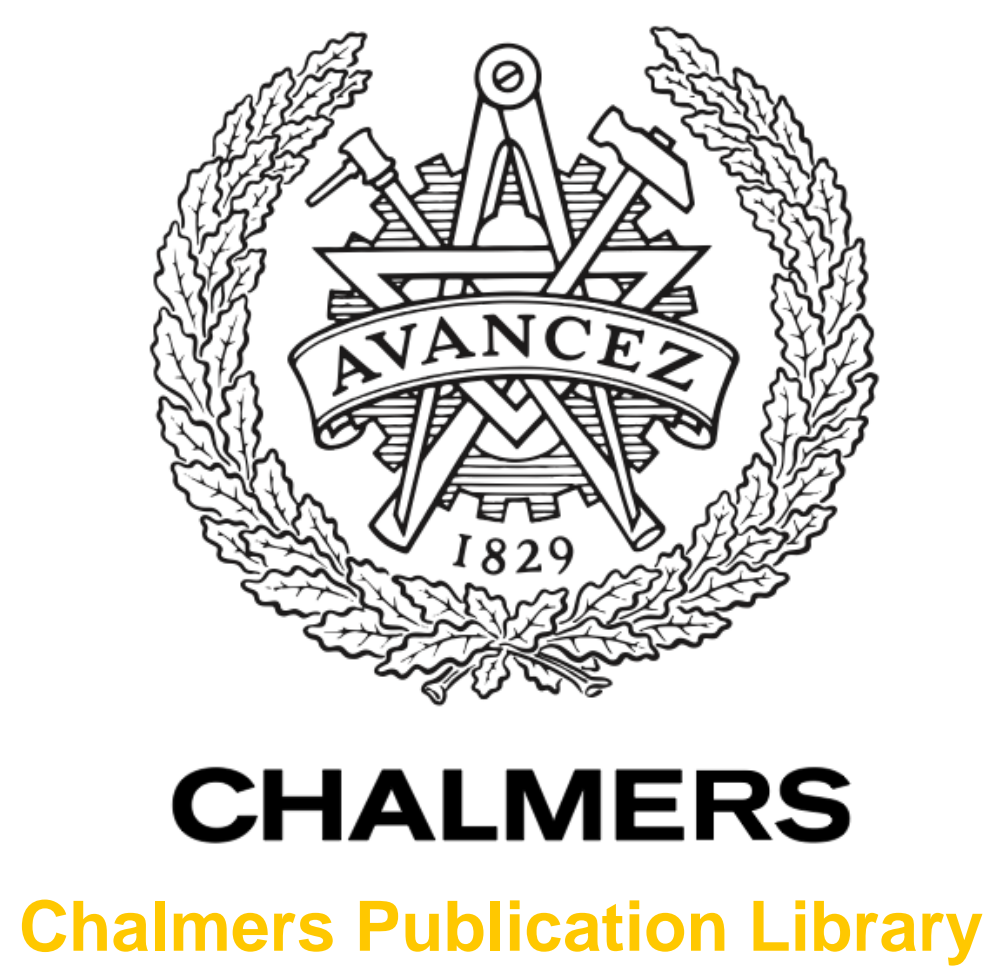

Energy Efficiency of VCSELs in the Context of Short-Range Optical Links.

This document has been downloaded from Chalmers Publication Library (CPL). It is the author's version of a work that was accepted for publication in:

IEEE Photonics Technology Letters (ISSN: 1041-1135)

Citation for the published paper:

Szczerba, K. ; Westbergh, P. ; Gustavsson, J. et al. (2015) "Energy Efficiency of VCSELs in the Context of Short-Range Optical Links.". IEEE Photonics Technology Letters, vol.

Pending(Pending), pp. Pending.

Downloaded from: http://publications.lib.chalmers.se/publication/219214

Notice: Changes introduced as a result of publishing processes such as copy-editing and formatting may not be reflected in this document. For a definitive version of this work, please refer to the published source. Please note that access to the published version might require a subscription.

Chalmers Publication Library (CPL) offers the possibility of retrieving research publications produced at Chalmers University of Technology. It covers all types of publications: articles, dissertations, licentiate theses, masters theses, conference papers, reports etc. Since 2006 it is the official tool for Chalmers official publication statistics. To ensure that Chalmers research results are disseminated as widely as possible, an Open Access Policy has been adopted.

The CPL service is administrated and maintained by Chalmers Library. 


\title{
Energy Efficiency of VCSELs in the Context of Short-Range Optical Links.
}

\author{
Krzysztof Szczerba, Member, IEEE, Petter Westbergh, Member, IEEE, Johan S. Gustavsson, \\ Magnus Karlsson, Senior Member, IEEE, Peter A. Andrekson, Fellow, IEEE, and Anders Larsson, Fellow, IEEE.
}

\begin{abstract}
We present results from an investigation of the energy efficiency of VCSELs under large signal modulation. We show that the most important factor influencing the energy consumption of the VCSELs is the required optical modulation amplitude, which drives other VCSEL design requirements. The required optical modulation amplitude also depends on the optical link design. Through this dependence it is possible to better understand the energy consumption of complete shortrange optical links.
\end{abstract}

Index Terms-optical communication, VCSEL, energy efficiency, datacom, optical modulation amplitude, energy consumption, optical link, optical interconnect.

\section{INTRODUCTION}

$\mathbf{S}$ HORT-range (below $300 \mathrm{~m}$ ) optical links in datacom applications are typically built using directly modulated low-cost vertical cavity surface emitting lasers (VCSELs), operating at the wavelength of $850 \mathrm{~nm}$. Multimode fiber (MMF) is usually used as the transmission medium. Data transmission is done by intensity modulation and direct detection (IM/DD).

VCSELs offer several advantages in this type of link. They can be directly modulated at very high data rates, recently demonstrated as high as 57 Gbps using on-off keying (OOK) without equalization [1] and 71 Gbps using OOK with equalization [2]. VCSELs are also energy efficient, for example the energy consumption in [1] was $510 \mathrm{fJ} / \mathrm{bit}$ at $57 \mathrm{Gbps}$ and $340 \mathrm{fJ} / \mathrm{bit}$ at $50 \mathrm{Gbps}$ and in [2] it was reported to be $260 \mathrm{fJ} / \mathrm{bit}$ at $71 \mathrm{Gbps}$. At lower bit-rates even lower energy consumption is reported, e.g. $140 \mathrm{fJ} / \mathrm{bit}$ at $34 \mathrm{Gbps}$ [3], $108 \mathrm{fJ} / \mathrm{bit}$ at $40 \mathrm{Gbps}$ [4] and $56 \mathrm{fJ} / \mathrm{bit}$ at $25 \mathrm{Gbps}$ [5]. Link energy consumption of $1 \mathrm{pJ} / \mathrm{bit}$ at $25 \mathrm{~Gb} / \mathrm{s}$ and $2.7 \mathrm{pJ} / \mathrm{bit}$ at $35 \mathrm{~Gb} / \mathrm{s}$ for links with $850 \mathrm{~nm}$ VCSELs are reported in [6] and $1.1 \mathrm{pJ} / \mathrm{bit}$ energy consumption at $26 \mathrm{~Gb} / \mathrm{s}$ for a link using a $1060 \mathrm{~nm}$ VCSEL in [7].

It was shown that a reduction in oxide aperture size results in a reduction of energy consumption per bit [8], because the $D$-factor is inversely proportional to the oxide aperture size. The resonance frequency is linearly dependent on the $D$-factor and therefore a VCSELs with smaller oxide aperture will reach higher bandwidths at low bias currents.

The oxide aperture also influences the maximum output

This work was financially supported by the Knut and Alice Wallenberg Foundation and the Swedish Foundation for Strategic Research.

K. Szczerba, P. Westbergh, J. S. Gustavsson, M. Karlsson, P. A. Andrekson and A. Larsson are with the Department of Microtechnology and Nanoscience, Chalmers University of Technology, Göteborg, Sweden (e-mail: krzysztof.szczerba@chalmers.se).

Manuscript received Month Day, 2015; Month Day, 2015.

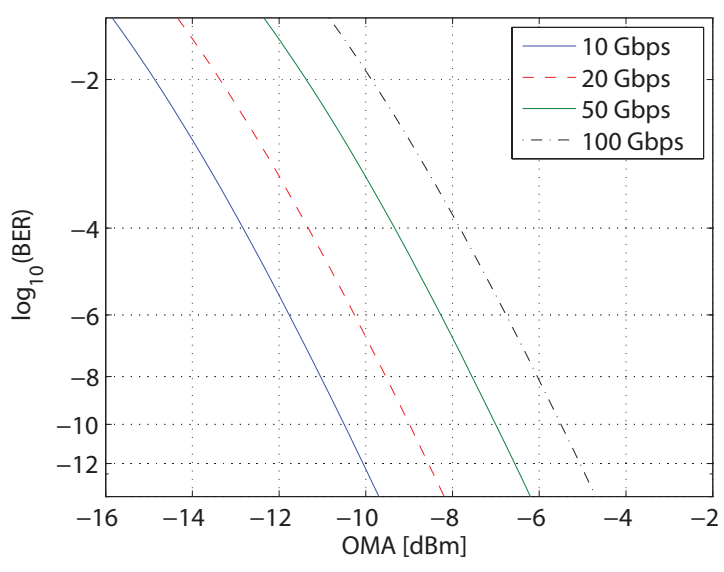

Fig. 1: BER as a function of OMA at the receiver for an IM/DD system with OOK, assuming a photodiode responsivity of $0.4 \mathrm{~A} / \mathrm{W}$

power of the VCSEL. Reduced oxide aperture diameter increases the VCSEL resistance which leads to thermal roll-over at lower current and optical output power. Therefore, reduced oxide aperture diameter results in reduced maximum optical output power.

For successful data transmission, the bit error rate (BER) must be sufficiently low. The BER depends on the signal-tonoise ratio (SNR), which depends on the optical modulation amplitude (OMA) and noise in the receiver. Therefore, the $B E R$ requirement and the receiver performance give a lower limit on the OMA.

In this letter we present the results of an experimental investigation of the relations between VCSEL oxide aperture diameter, modulation bandwidth, OMA at the VCSEL output and VCSEL energy consumption.

\section{OMA REQUIREMENTS IN SHORT-RANGE OPTICAL LINKS}

In a typical optical link without forward error correction (FEC) the bit error rate (BER) for "error-free" transmission must be below $10^{-12}$. In general, the BER is a function of the SNR. In IM/DD systems, the main noise sources are the thermal noise in the receiver, shot noise and relative intensity noise (RIN) of the laser. For simplicity it is assumed that the system is dominated by thermal noise with variance $\sigma$. RIN will cause a sensitivity penalty, which can be calculated as given in [9, Ch. 5.4.2]. For RIN below $-145 \mathrm{dBm} / \mathrm{Hz}$ and 
received optical power below $0 \mathrm{dBm}$ this penalty is negligible. In a direct detection system the electrical signal current $I$ is proportional to the optical signal power $P$ through the photodiode responsivity $R_{P D}$ as $I=R_{P D} P$. For OOK the BER at the receiver is $[9, \mathrm{Ch}$. 5.3.1]

$$
\mathrm{BER}=\frac{1}{4}\left[\operatorname{erfc}\left(\frac{I_{1}-I_{t h}}{\sigma \sqrt{2}}\right)+\operatorname{erfc}\left(\frac{I_{t h}-I_{0}}{\sigma \sqrt{2}}\right)\right],
$$

where $I_{0}, I_{1}$ and $I_{t h}$ are currents at, respectively, bit 0 , bit 1 and the decision threshold. The optical power levels corresponding to 0 and 1 are denoted $P_{0}, P_{1}$ and OMA $=P_{1}-P_{0}$. If the decision threshold is placed half way in between the levels 0 and 1 then (1) can be simplified to

$$
\mathrm{BER}=\frac{1}{2} \operatorname{erfc}\left(\frac{R_{P D} \mathrm{OMA}}{\sigma 2 \sqrt{2}},\right) .
$$

The responsivity depends on wavelength and receiver. The noise variance depends on bandwidth (and therefore bit-rate), temperature, noise figure and load resistance. The OMA at the receiver has to be large enough to achieve the required BER. An example theoretical BER as a function of the OMA at the receiver for a system dominated by thermal noise, with $R_{P D}=0.4 \mathrm{~A} / \mathrm{W}, \mathrm{RIN}=-145 \mathrm{~dB} / \mathrm{Hz}, 5 \mathrm{~dB}$ receiver noise figure and for bit-rates in the range between $10 \mathrm{Gbps}$ and $100 \mathrm{Gbps}$ is shown in Fig. 1. Note that this is best-case BER, assuming thermal noise dominated system. The OMA at the VCSEL output has to be larger because of coupling and fiber losses, intersymbol interference penalty, mode partition noise, RIN, and performance degradation due to ageing. The link budgets of short-range optical links are typically around $8 \mathrm{~dB}$ as specified in the Ethernet and Infiniband standards [10], [11]. The theoretical required transmitter OMA required to reach BER below $10^{-12}$, based on results in Fig. 1 and including $8 \mathrm{~dB}$ link budget is shown in Table I, including assumed noise variance. There are no VCSELs capable of supporting $100 \mathrm{Gbps}$ OOK tranmission at the time of writing, however, it is expected that $100 \mathrm{Gbps}$ will be achievable in the future.

From the system perspective, a more power efficient VCSEL is the one that delivers a required OMA at a energy to data ratio (EDR), defined as in [8]. The EDR is calculated from the DC and RF input power and the data-rate. Another commonly used figure of merit is the heat to data ratio (HDR) which is the ratio between the dissipated power (difference between the electrical input power and optical output power) and the data-rate [8].

\section{Measurements}

The EDR was measured as a function of the following paramters: OMA, oxide aperture diameter and extinction ratio

TABLE I: Reqired launch OMA for selected bit-rates, assuming OOK and $8 \mathrm{~dB}$ link budget.

\begin{tabular}{|l|l|l|l|}
\hline $\begin{array}{l}\text { Bit-rate } \\
{[\mathrm{Gbps}]}\end{array}$ & $\begin{array}{l}\text { Required } \\
\text { OMA }[\mathrm{dBm}]\end{array}$ & $\begin{array}{l}\text { Required } \\
\text { OMA }[\mathrm{mW}]\end{array}$ & $\begin{array}{l}\text { noise } \\
\text { variance }[\mu \mathrm{A}]\end{array}$ \\
\hline 10 & -2 & 0.63 & 2.8 \\
\hline 20 & -0.5 & 0.89 & 3.8 \\
\hline 50 & 1.5 & 1.4 & 6.3 \\
\hline 100 & 3 & 2 & 8.9 \\
\hline
\end{tabular}
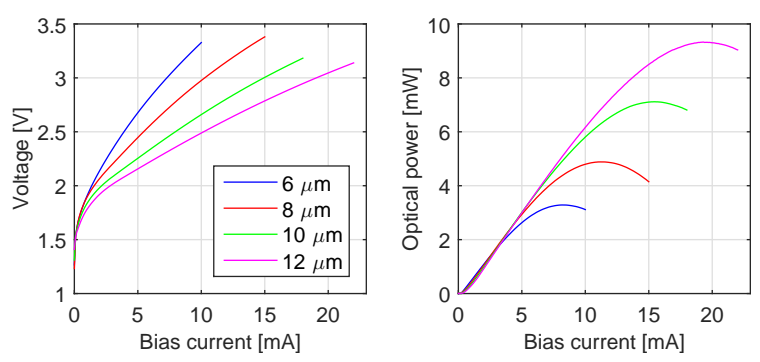

Fig. 2: Static characteristics of the VCSELs used in the measurements. The legend gives the oxide aperture diameters.

$\mathrm{ER}=\mathrm{P}_{1} / \mathrm{P}_{0}$. The EDR includes both the DC and RF power fed into the VCSEL.

Four VCSELs with the following oxide aperture diameters were used: $6,8,10$ and $12 \mu \mathrm{m}$. The VCSELs were of a design described in detail in [12]. The design uses strained InGaAs quantum wells in the active region for high differential gain and binary AlAs in the bottom DBR to facilitate heat transfer. Two confining oxide apertures were incorporated to reduce the device capacitance. The static characteristics of the VCSELs are shown in Fig. 2. It can be seen that devices with smaller aperture diameters reach lower maximum optical output power. Small aperture devices have also lower threshold currents, but the threshold currents are all below $1 \mathrm{~mA}$ and thus the differences are hardly visible in Fig. 2. For each VCSEL the input impedance, frequency response and their dependency on bias current were also measured. The frequency response was used to calculate achievable bit-rate, assuming that data rate of 1.43 times the $-3 \mathrm{~dB}$ bandwidth is possible with negligible intersymbol interference. Equalization was ruled out to constrain the problem.

The test setup to measure the EDR and HDR comprised an arbitrary waveform generator (AWG) with $5 \mathrm{GHz}$ analog bandwidth as a source of the RF signal and a DC current source. The DC bias and RF signals were combined in a bias-T and fed to the VCSEL, which was connected using a microwave probe. The optical output of the VCSEL was coupled to a $2 \mathrm{~m}$ MMF patchcord through a lens package. The signal was detected with a $25 \mathrm{GHz}$ photodiode and analyzed on an oscilloscope. The AWG was programmed with a $1 \mathrm{Gbps}$ OOK pattern because it could be supported by all VCSELs even at low bias currents and simplified automatic eye opening measurement on the oscilloscope in presence of e.g. ringing due to resonance peaks. The RF input power from to the VCSEL was calculated from the AWG output power and reflection due to impedance mismatch, similarly to [8]. For each VCSEL the bias current was swept in a range between the threshold current and 1.3 times the thermal roll-over current and the modulating signal amplitude in the range between 0.5 and $1 \mathrm{~V}$, which was limited by the AWG. The OMA was automatically measured from the eye diagrams on the oscilloscope. The average optical power was measured from the photodiode current monitor and compared with the known optical output power at a given bias point for each VCSEL (as given in fig. 2) in order to calculate the link loss, which was around $3 \mathrm{~dB}$, but varied within $0.5 \mathrm{~dB}$ with bias current 


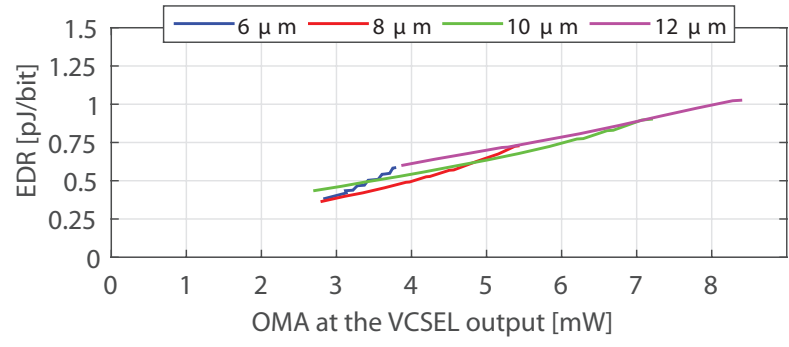

(a)

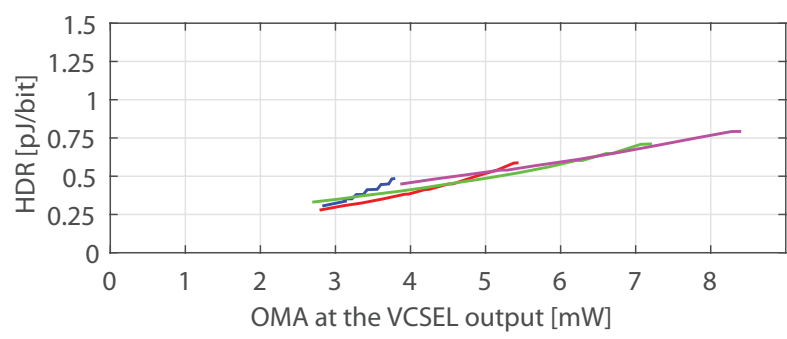

(b)

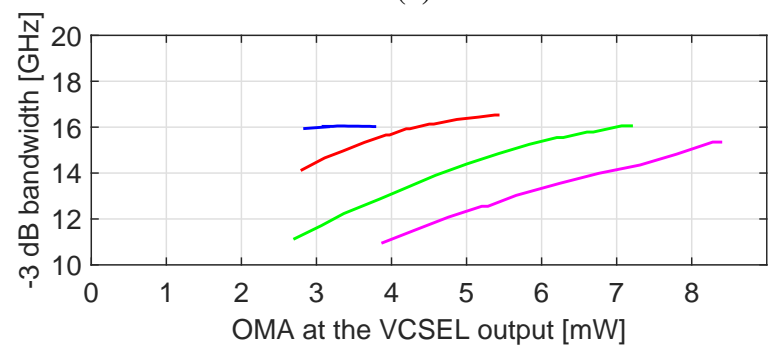

(c)

Fig. 3: EDR as a function of the OMA (a), HDR as a function of the OMA (b) and $-3 \mathrm{~dB}$ bandwidth at a given OMA (c) at $10 \mathrm{~dB}$ ER for VCSELs with $6,8,10$ and $12 \mu \mathrm{m}$ oxide aperture diameters (given in the legend).

and oxide aperture. The loss was deembedded to calculate the OMA at the VCSEL output.

\section{RESULTS}

The DC power, which was in the range 5 to $50 \mathrm{~mW}$, depending on the bias, contributed the majority (at least $87 \%$ ) of the total input power. The $-3 \mathrm{~dB}$ bandwidth was used to calculate the EDR, assuming data rate of 1.43 time the $-3 \mathrm{~dB}$ bandwidth. The EDR, the HDR and the corresponding $-3 \mathrm{~dB}$ bandwidth for the four VCSELs, plotted against the OMA, are shown in Figs. 3-5 at ER of $10 \mathrm{~dB}, 6 \mathrm{~dB}$ and $3 \mathrm{~dB}$, respectively.

\section{A. $10 \mathrm{~dB} E R$}

At the $10 \mathrm{~dB}$ ER the EDR and the HDR are nearly linearly dependent on the OMA, decreasing with decreasing OMA, as illustrated in Fig. 3. The ratio of HDR to the input energy per bit is in between $77 \%$ and $87 \%$, depending on the bias point and the aperture size. The EDR and HDR at a given OMA value do not depend strongly on the oxide aperture size. The modulation bandwidth, on the other hand, depends strongly on the oxide aperture diameter is the modulation bandwidth. At

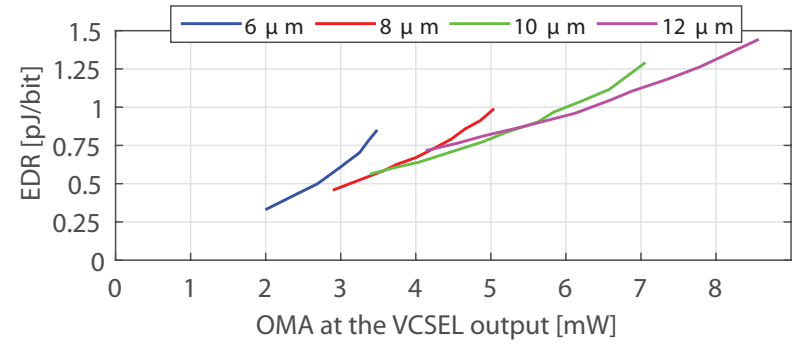

(a)

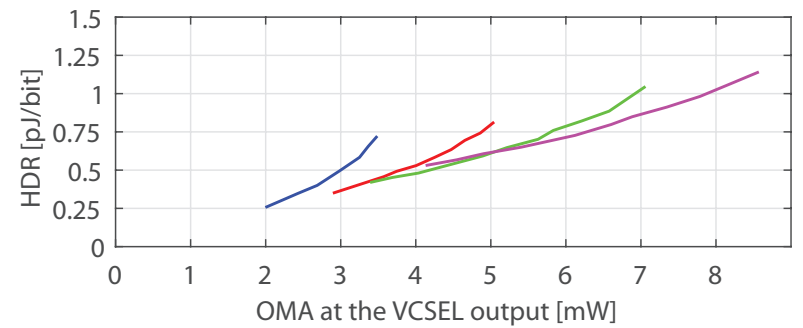

(b)

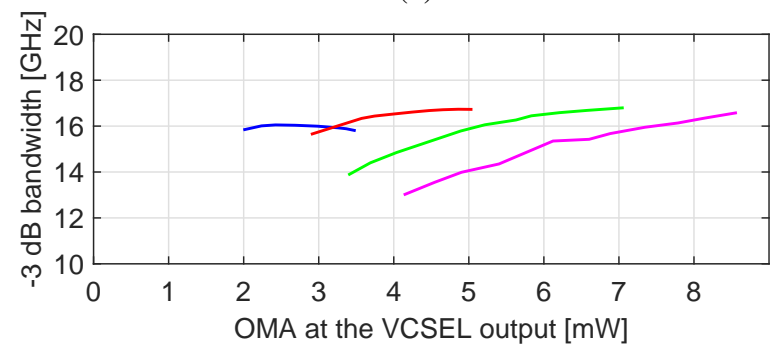

(c)

Fig. 4: Same as in Fig. 3, at $6 \mathrm{~dB}$ ER.

the same OMA, small aperture VCSELs achieve significantly higher bandwidths. The increased bandwidth should reduce the energy consumption per bit, but it is counteracted by increased impedance of the small aperture VCSELs.

\section{B. $6 d B E R$}

Reducing the ER from 10 to $6 \mathrm{~dB}$ results in increased EDR and HDR, as shown in Fig. 4. The increase is between $25 \%$ and $30 \%$. The increment is higher when the VCSELs have to be biased closer to thermal roll-over to reach the required OMA. This makes the energy consumption per bit non-linearly dependent on the OMA. If high OMA is required, then from energy efficiency point of view it is better to switch to VCSELs with larger apertures than to drive small VCSELs at high currents.

\section{C. $3 d B$ ER}

A further reduction in the $\mathrm{ER}$ to $3 \mathrm{~dB}$ results in further increase in energy consumption, as shown in Fig. 5. Increase in OMA results in faster than linear increase in energy consumption. At lower ERs, larger aperture diameters are necessary to reach a given OMA. Compared to the $10 \mathrm{~dB}$ $\mathrm{ER}$, the EDR and HDR are at least doubled for given OMA. Low ERs contribute significantly to energy consumption and high ERs are needed for energy efficient operation. For each 


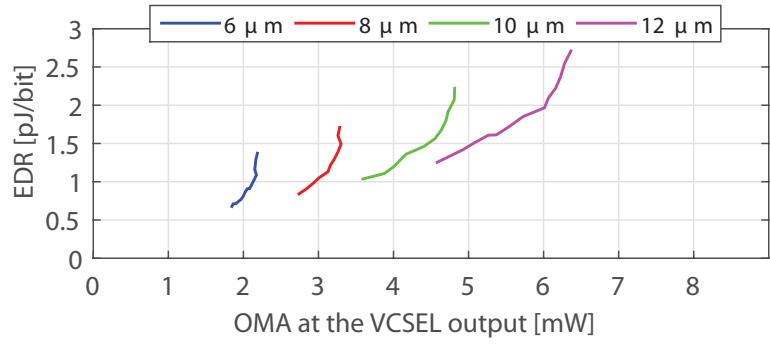

(a)

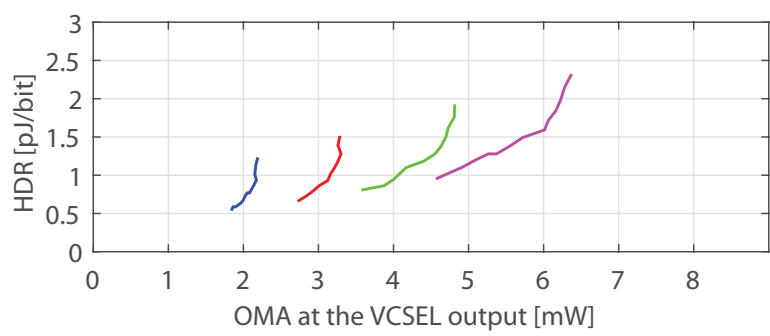

(b)

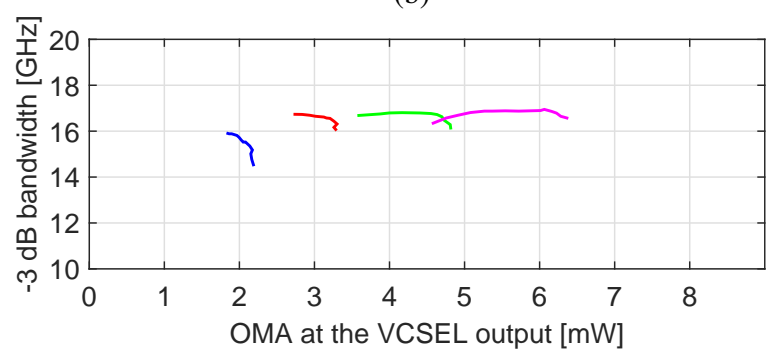

(c)

Fig. 5: Same as in Fig. 3, at $3 \mathrm{~dB}$ ER.

OMA value there is an optimal aperture size which maximizes the modulation bandwidth.

\section{Link and laser co-optimization}

It is known that smaller VCSEL oxide apertures are better [8] for high bandwidth and good energy efficiency, but the requirement on minimum OMA constrains the minimum oxide aperture diameter. The tested lasers were able to deliver OMA in excess of the best case scenario requirements given in Table I. This means that the apertures and energy consumption could be reduced, until the lower limit of the OMA is reached.

In order to reduce the energy consumption per bit, the OMA requirement itself needs to be reduced. The ER should be kept high, but $10 \mathrm{~dB}$ may may problematic because of chirp and modulation close to the threshold, therefore $6 \mathrm{~dB}$ may be a reasonable choice.

The transmitter OMA requirement can be reduced by FEC and reduction of the link budget. The FEC can reduce the OMA requirement, but it will introduce additional energy consumption in the encoders and decoders. Reduction of the link budget means reduction of the penalty allocations. This requires careful review of the current assumptions about e.g. RIN, performance loss due to ageing and so forth.

There are also other developments in the field, such as multilevel modulation formats. The appeal of multilevel formats is that the bit-rates can be increased, but at the same time the OMA requirement will increase. This can be mitigated to some extent by the use of FEC. However, for proper systemlevel optimization, the entire transmitter, including the driver amplifiers has to be included in the analysis.

\section{Conclusions}

We have demonstrated, that the energy consumption per bit in high-speed VCSELs is depended on the required OMA. In the limit of high ER the energy per bit is linearly dependent on the OMA. The oxide aperture size affects primarily the modulation bandwidth. Reduced oxide aperture sizes allow a larger modulation bandwidth at lower OMA and bias current, but this effect is countered by accompanying increase of the VCSEL impedance. At low ER a larger bias current is necessary to reach the same OMA. Consecutively, thermal effects begin to play important role and the energy consumption per bit increases non-linearly with the OMA.

The OMA requirement is driven by the link design, which can be optimized to reduce the total energy consumption. An example of optimization problem is introduction of FEC. The FEC will reduce the energy consumption in the VCSEL and the driver, but it will add power consumption in the encoder and decoder circuits.

\section{REFERENCES}

[1] P. Westbergh, E. P. Haglund, E. Haglund, R. Safaisini, J. S. Gustavsson, and A. Larsson, "High-speed $850 \mathrm{~nm}$ VCSELs operating error free up to 57 Gbit/s," Electron. Lett., vol. 49, no. 16, pp. 1021-1023, Aug. 2013.

[2] D. M. Kuchta, A. V. Rylyakov, F. E. Doany, C. L. Schow, J. E. Proesel, C. W. Baks, P. Westbergh, J. S. Gustavsson, and A. Larsson, "A $71 \mathrm{~Gb} / \mathrm{s}$ NRZ modulated $850 \mathrm{~nm}$ VCSEL-based optical link," Accepted to IEEE Photon. Technol. Lett., 2015.

[3] J.-W. Shi, J.-C. Yan, J.-M. Wun, J. Chen, and Y.-J. Yang, "Oxiderelief and zn-diffusion 850-nm vertical-cavity surface-emitting lasers with extremely low energy-to-data-rate ratios for $40 \mathrm{Gbit} / \mathrm{s}$ operations," IEEE J. Sel. Topics Quantum Electron., vol. 19, no. 2, pp. 7900208 7900 208, Mar. 2013.

[4] P. Wolf, P. Moser, G. Larisch, H. Li, J. A. Lott, and D. Bimberg, "Energy efficient $40 \mathrm{Gbit} / \mathrm{s}$ transmission with $850 \mathrm{~nm}$ VCSELs at $108 \mathrm{fJ} / \mathrm{bit}$ dissipated heat," Electron. Lett., vol. 49, no. 10, pp. 666-667, May 2013.

[5] P. Moser, J. A. Lott, P. Wolf, G. Larisch, H. Li, N. N. Ledentsov, and D. Bimberg, "56 fJ dissipated energy per bit of oxide-confined $850 \mathrm{~nm}$ VCSELs operating at $25 \mathrm{Gbit} / \mathrm{s}, "$ Electronics Letters, vol. 48, no. 20, pp. 1292-1294, Sept. 2012.

[6] J. E. Proesel, B. G. Lee, C. W. Baks, and C. L. Schow, "35-Gb/s VCSEL-based optical link using 32-nm SOI CMOS circuits," in Proc. OFC/NFOEC, Mar. 2013, paper OM2H.2.

[7] J. B. Heroux, T. Kise, M. Funabashi, T. Aoki, C. L. Schow, A. V. Rylyakov, and S. Nakagawa, "Energy-efficient 1060-nm optical link operating up to $28 \mathrm{~Gb} / \mathrm{s}$," J. Lightw. Technol., vol. 33, no. 4, pp. 733-740, Feb. 2015.

[8] P. Moser, J. A. Lott, P. Wolf, G. Larisch, H. Li, N. N. Ledentsov, and D. Bimberg, "Impact of the aperture diameter on the energy efficiency of oxide-confined $850 \mathrm{~nm}$ high speed VCSELs," Proc. SPIE, vol. 8639, pp. 86390V-86390V-8, Mar. 2013.

[9] G. Agrawal, Lightwave technology: telecommunication systems. WileyInterscience, 2005.

[10] IEEE Standard for Ethernet, 2012, no. IEEE Standard 802.3-2012.

[11] Infiniband Trade Association, InfiniBandTM Architecture Specification Volume 2 Release 1.3, Beaverton, OR97006, USA, 2012.

[12] P. Westbergh, J. S. Gustavsson, B. Kögel, Å. Haglund, and A. Larsson, "Impact of photon lifetime on high-speed VCSEL performance," IEEE J. Sel. Topics Quantum Electron., vol. 17, no. 6, pp. 1603-1613, Nov. 2011. 